\title{
Entropy-Monotone Scheme for Nonlinear Scalar Conservation Laws
}

\author{
Li Xiao, Rongsan Chen ${ }^{*}$, Miao Wang and Xia Zhang \\ School of Mathematics and Physics, China University of Geosciences, Wuhan 430074, P. R. China \\ ${ }^{*}$ Corresponding author
}

\begin{abstract}
The Entropy-TVD scheme was developed for the nonlinear scalar conservation laws in [1].The scheme with step reconstruction simultaneously compute the two numerical entities, the numerical solution and the numerical entropy, and numerical examples show that the scheme provides a super-convergence rate. In this paper, we present an Entropy-Monotone scheme with linear reconstruction for the non-linear scalar conservation laws. The GRP fluxes are used to compute numerical fluxes. Numerical tests show that the convergent rates of EntropyMonotone scheme are more stable than Entropy-TVD scheme and Entropy-Monotone scheme has better resolution of shocks and corners of rarefaction waves.
\end{abstract}

Keywords-entropy-monotone scheme; GRP numerical fluxes; high resolution

\section{INTRODUCTION}

As we all know, the solutions of the hyperbolic conservation laws might develop discontinuity even if the initial conditions are very smooth. Once encounter discontinuity, the classical solutions are no longer satisfied in the classic sense but are satisfied in the weak sense. Although weak solutions allow for discontinuities, they may not be unique in general. Thus, in order to obtain unique physical solution, it need impose an extra entropy condition. Many schemes does not satisfy the entropy condition, such as the famous Roe scheme [2]. A lot of entropy fix techniques were developed to modify the Roe scheme, see [3], [4]. Entropyconservative schemes and entropy-stable schemes were presented, see [5], [6], [7], [8].

Recently, Li, Wang and Mao presented a new type of finite volume scheme for numerical simulations of evolution partial differential equations, see [9], [10], [11], [12], [13], [14] and [1]. Different from the traditional Godunov-type schemes, the scheme involves two numerical entities, numerical solution and numerical entropy, and maintains the conservation of both the solution and the entropy. The reconstruction of the schemes are based on physical relations. The entropy scheme was firstly presented by $\mathrm{Li}$ and Mao the entropy scheme for the linear advection equation in [10]. However, when computing discontinuous solutions, the entropy scheme produced spurious oscillations near discontinuities. To fix this problem, in [12], Li and Mao extended the entropy scheme for the linear advection equation to the Euler equations of compressible gas dynamics and proposed the so-called Entropy-Ultra-Bee scheme which is the marriage of the entropy scheme and the Ultra-bee scheme. The Entropy-Ultra-
Bee scheme is of an oscillation-free scheme which has good resolution in smooth regions as well as near discontinuities. In [14], Cui and Mao revealed that the entropy scheme had an error self-canceling mechanism and showed that the scheme was super convergent.

In [1], Chen and Mao developed the Entropy-TVD scheme for the non-linear scalar conservation laws. Chen and Mao gave some good properties of the scheme, such as feasibility, TVD and entropy condition satisfaction. But the convergent rates of Entropy-TVD scheme are not stable, especially the convergence rates of L1 swing up and down in 1.

In this paper, we present an Entropy-Monotone scheme. Using linear reconstruction, a generalized Riemann problem is solved for computing the numerical fluxes. We compute the numerical solution in conservative fashion; however, we compute the numerical entropy in non conservative fashion because there maybe encounter discontinuity. The feasibility of the scheme is proved and the entropy condition is satisfied.

The organization of this paper is as follows. Section I is the introduction. In Section II, the Entropy-Monotone scheme is described. In Section III, we present several numerical examples, which show that the convergent rates of EntropyMonotone scheme are more stable than Entropy-TVD scheme and Entropy-Monotone scheme has better resolution of shocks and corners of rarefaction waves. Finally, the conclusion is given in section 5 .

\section{ENTROPY-MONOTONE SCHEME FOR NONLINEAR ADVECTION EQUATIONOF}

We consider the following initial value problem of the nonlinear advection equation

$$
\left\{\begin{array}{l}
u_{t}+f(u)_{x}=0, \\
u(x, 0)=u_{0}(x),
\end{array}\right.
$$

where $u$ and $f(u)$ are scalars. For simplicity, we assume $f(u)$ is a strict convex function of $u$.

A physically meaningful solution require (1) satisfies the entropy condition 


$$
U(u)_{t}+F(u)_{x} \leq 0 .
$$

where $F(u)$ is the entropy and $(U(u) ; F(u))$ is an entropy pair for the system. The equality in (2) holds in smooth region, where the total entropy is conserved. But it decreases across a shock.

For simplicity, we assume that the grid points $x_{i}$ are uniformly distributed with the cell size $d x$ and we also denote the cells by $\left(x_{j-1 / 2}, x_{j+1 / 2}\right)$. We use $\tau$ to denote the time increment. The Entropy-Monotone scheme involves two numerical entities, the numerical solution un and the numerical entropy $U^{n} \cdot u^{n}$ and $U^{n}$ are defined as

$$
u_{j}^{n} ; \frac{1}{h} \int_{x_{j-1 / 2}}^{x_{j+1 / 2}} u\left(x, t_{n}\right) d x
$$

and

$$
U_{j}^{n} ; \frac{1}{h} \int_{x_{j-1 / 2}}^{x_{j+1 / 2}} U\left(x, t_{n}\right) d x .
$$

The Entropy-Monotone scheme contains the following three steps:

i) Reconstruction. We use a linear function in each cell to obtain the reconstruction,

$$
R\left(x ; u_{n} ; U_{n}\right)=u_{j}^{n}+s_{j}^{n}\left(x-x_{j}\right)
$$

Where $s_{j}^{n}$ is the slope of the linear reconstruction. It is not difficult to obtain

$$
\frac{1}{h} \int_{x_{j-1 / 2}}^{x_{j+1 / 2}} R\left(x ; u^{n}, U^{n}\right) d x=u_{j}^{n} .
$$

In order to compute slope $s_{j}^{n}$, we require an entropy slope $S_{j}^{n, e}$ satisfies

$$
\frac{1}{h} \int_{x_{j-1 / 2}}^{x_{j+1 / 2}} U\left(R\left(x ; u^{n}, U^{n}\right)\right) d x=U_{j}^{n} .
$$

Eq. (7) means that the entropy cell-average of the reconstructed solution is equal to the numerical entropy in the cell.

We then compute a monotone slope $s_{j}^{n \text {,mon }}$ by a monotone limiter on the reconstruction, see [15],

$$
s_{j}^{n, \text { mon }}=\min \bmod \left(\alpha \frac{u_{j}^{n}-u_{j-1}^{n}}{h}, \alpha \frac{u_{j+1}^{n}-u_{j}^{n}}{h}\right) .
$$

the parameter $\alpha \in[0,2]$. The slope is then chose as the one smaller in absolute value of $s_{j}^{n, e}$ and $s_{j}^{n, m o n}$ with the sign of

$$
\begin{aligned}
& \left(u_{j+1}^{n}-u_{j-1}^{n}\right) \\
& \quad s_{j}^{n}=\operatorname{sgn}\left(u_{j+1}^{n}-u_{j-1}^{n}\right) \min \left(\left|s_{j}^{n, e}\right|,\left|s_{j}^{n, \text { mon }}\right|\right) .
\end{aligned}
$$

ii) Evolution. We solve the following generalized initial value problem

$$
\left\{\begin{array}{l}
v_{t}+f(v)_{x}=0, \quad t_{n}<t \leq t_{n+1} \\
v(x, 0)=R\left(x ; u^{n} ; U^{n}\right)
\end{array}\right.
$$

and obtain the solution $v(x ; t)$ over the time interval $\left[t_{n} ; t_{n+1}\right]$.

iii) Cell-averaging. The numerical solution $u_{j}^{n+1}$ and the numerical entropy $U_{j}^{n+1}$ at $t_{n+1}$ is computed as

$$
u_{j}^{n+1}=\frac{1}{h} \int_{x_{j-1 / 2}}^{x_{j+1 / 2}} v\left(x, t_{n+1}\right) d x,
$$

and

$$
U_{j}^{n+1}=\frac{1}{h} \int_{x_{j-1 / 2}}^{x_{j+1 / 2}} U\left(v\left(x, t_{n+1}\right)\right) d x
$$

$u_{j}^{n+1}$ can also be computed in the following flux form,

$$
u_{j}^{n+1}=u_{j}^{n}-\lambda\left(\hat{f}_{j+1 / 2}^{n}-\hat{f}_{j-1 / 2}^{n}\right) \text {, }
$$

where

$$
\hat{f}_{j+1 / 2}^{n}=\frac{1}{\tau} \int_{t_{n}}^{t_{n+1}} f\left(v\left(x_{j+1 / 2}, t\right)\right) d t .
$$

$U_{j}^{n+1}$ can also be computed in the following flux form,

$$
\begin{gathered}
U_{j}^{n+1}=\frac{1}{h} \int_{x_{j-1 / 2}}^{x_{j+1 / 2}} U\left(R\left(x ; u^{n}, U^{n}\right)\right) d x-\lambda\left(\hat{F}_{j+1 / 2}^{n}-\hat{F}_{j-1 / 2}^{n}\right) \\
+\lambda \sum_{i} D_{j}^{n, i}
\end{gathered}
$$

where

$$
\hat{F}_{j+1 / 2}^{n}=\frac{1}{\tau} \int_{t_{n}}^{t_{n+1}} \mathrm{~F}\left(v\left(x_{j+1 / 2}, t\right)\right) d t
$$

and $D_{j}^{n, i}$ is the entropy decrease of $v(x, t)$ across the ith shock in the grid cell $\left(x_{j-1 / 2}, x_{j+1 / 2}\right) \times\left(t_{n}, t_{n+1}\right)$,see [1] . 
We then choose $U(u)=u^{2}$ is computed as

$$
s_{j}^{n, e}=\operatorname{sgn}\left(u_{j+1}^{n}-u_{j-1}^{n}\right) \sqrt{\frac{12\left(U_{j}^{n}-\left(u_{j}^{n}\right)^{2}\right)}{h^{2}}}
$$

$\hat{f}_{j+1 / 2}^{n}$ and $\hat{F}_{j+1 / 2}^{n}$ is computed as GRP numerical fluxes, see [15].

\section{NUMERICAL EXPERIMENTS}

In this section, we show some results of EntropyMonotone scheme with GRP numerical fluxes for several examples. We consider Eq. (1) to be the Burgers equation. The CFL number is taken to be $0: 2$ and is taken to be 2 . To test our scheme and comprise with Entropy-TVD scheme, recompute of the numerical examples in [1]. The "New" stands for the numerical solution computed by the Entropy-Monotone scheme.

Example 1. The initial conditions are

$$
u_{0}(x)=\sin 2 \pi x, \quad 0 \leq x \leq 1
$$

with periodic boundary conditions at the two ends. When $t=0.1$ the solution is still smooth. This example is used to investigate the smooth solutions. The errors and numerical orders of accuracy are presented in Table I . It is seen from the table that the $L_{1}$ errors show a second order convergence rate, which indicates that the scheme is essentially secondorder accurate globally. However, the $L_{\infty}$ errors show a much lower, but still greater than 1 , convergence rate. The convergence rates of $L_{\infty}$ for Entropy-TVD scheme swing up and down in 1, and the errors of Entropy-TVD scheme is larger than Entropy-Monotone scheme, refer to [1]. The convergence rates of $L_{1}$ and $L_{\infty}$ of Entropy-Monotone scheme are stable.

TABLE I. EXAMPLE 1, NUMERICAL ERRORS AND CONVERGENCE RATES AT T $=2$

\begin{tabular}{|c|c|c|c|c|}
\hline cells & $L_{\infty}$ error & Rate & $L_{1}$ error & Rate \\
\hline 20 & $2.640 \mathrm{E}-002$ & - & $1.039 \mathrm{E}-002$ & - \\
\hline 40 & $1.315 \mathrm{E}-002$ & 1.004 & $3.103 \mathrm{E}-003$ & 1.744 \\
\hline 80 & $6.556 \mathrm{E}-003$ & 1.004 & $8.427 \mathrm{E}-004$ & 1.880 \\
\hline 160 & $3.272 \mathrm{E}-003$ & 1.002 & $1.990 \mathrm{E}-004$ & 2.082 \\
\hline 320 & $1.634 \mathrm{E}-003$ & 1.001 & $4.844 \mathrm{E}-005$ & 2.038 \\
\hline 640 & $8.167 \mathrm{E}-004$ & 1.000 & $1.174 \mathrm{E}-005$ & 2.044 \\
\hline 1280 & $4.082 \mathrm{E}-004$ & 1.000 & $2.820 \mathrm{E}-006$ & 2.058 \\
\hline
\end{tabular}

Example 2. The initial conditions are

$$
u_{0}(x)=\left\{\begin{array}{l}
1, \text { if } 0 \leq x<0.1 \\
2, \text { if } 0.1 \leq x<0.6 \\
1, \text { if } 0.6 \leq x<01
\end{array}\right.
$$

The computed solution by the Entropy-Monotone scheme are plotted at $t=0.2$ against the exact solution in Figure I . The computed results by Entropy-Monotone scheme are very comparable to the analytical solution. The computed results are oscillatory free in the vicinity of shock and the corner of rarefaction wave.

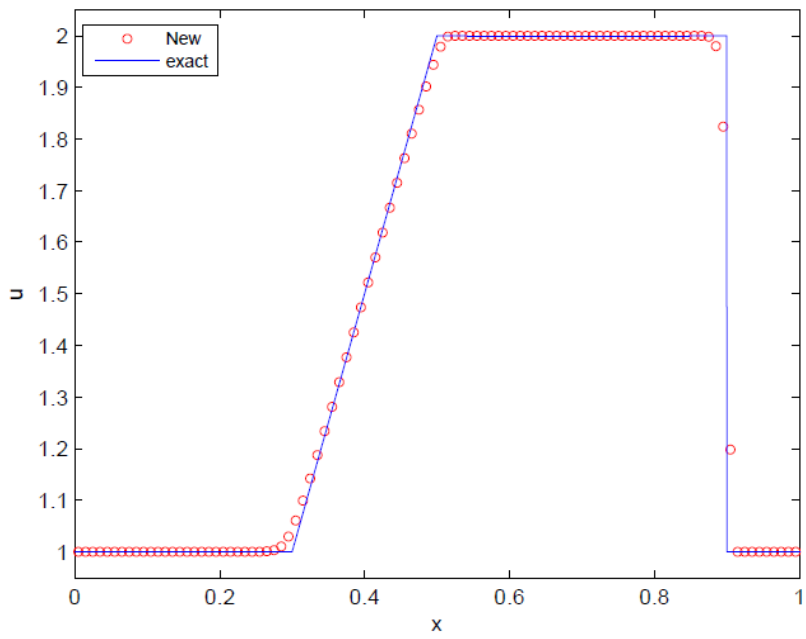

FIGURE I. NUMERICAL SOLUTION TO EXAMPLE 2 ON 100 CELLS $\mathrm{AT} \mathrm{T}=0: 2$

\section{ACKNOWLEDGMENT}

The research is supported by the National Natural Science Foundation of China No.11201436.

\section{REFERENCES}

[1] R. S. Chen, D.-K. Mao, Entropy-TVD scheme for nonlinear scalar conservation laws, J.Sci. Comput. 47 (2011) 150-169.

[2] P. L. Roe, Approximate riemann solvers, parameter vectors, and difference schemes, J.Comput. Phys. 43 (1981) 357-372.

[3] A. Harten, High resolution schemes for hyperbolic conservation laws, J. Comput. Phys.49 (1983) 357-393.

[4] A. Harten, On the symmetric form of systems of conservation laws with entropy, J.Comput. Phys. 49 (1983) 151-164.

[5] E. Tadmor, Numerical viscosity of entropy stable schemes for systems of conservation laws, Math. Comp. 49 (1987) 91-103.

[6] E. Tadmor, Entropy stability theory for difference approximations of nonlinear conservation laws and related time-dependent problems, Acta Numer. 12 (2003) 451-512.

[7] E. Tadmor, W. Zhong, Entropy stable approximations of navier-stokes equations with no artificial numerical viscosity, J. HYPERBOL. DIFFER. EQ. 3 (2006) 529-559.

[8] X. H. Chen, Y. F. Nie, J. H. Feng, X. Y. Luo, L. Cai, Self-adjusting entropy-stable scheme for compressible euler equations, Chin. Phys. B 24 (2015) 020202.

[9] Z. G. Wang, Finitie difference scsemes satisfying multiconservation laws for linear advection equations, Master's thesis, No. 1190399118086, Shanghai University (in Chinese). 
[10] H. X. Li, Entropy dissipating scheme for hyperbolic system of conservation laws in one space dimension, Doctoral thesis, No. 1190302820022, Shanghai University (in Chinese).

[11] Z. G. Wang, D.-K. Mao, Conservative difference scheme satisfying three conservation laws for linear advection equation, J. SHU (in Chinese) 6 (2006) 588-598(in Chinese).

[12] H. X. Li, Z. G.Wang, D.-K. Mao, Numerically neither dissipative nor compressive scheme for linear advection equation and its application to the Euler system, J. Sci. Comput.36 (2008) 285-331.

Y. F. Cui, D.-K. Mao, Numerical method satisfying the first two conservation laws for the Korteweg-de Vries equation, J. Comput. Phys. 227 (2007) 376-399.

[13] Y. F. Cui, D.-K. Mao, Error self-canceling of a difference scheme maintaining two conservationlaws for linear advection equation, Math. Comput. 81 (2012) 715-741.

[14] M. Ben-Artzi, J. Q. Li, G. Warnecke, A direct eulerian grp scheme for compressible fluid flows, J. Comput. Phys. 218 (2006) 19-43. 